
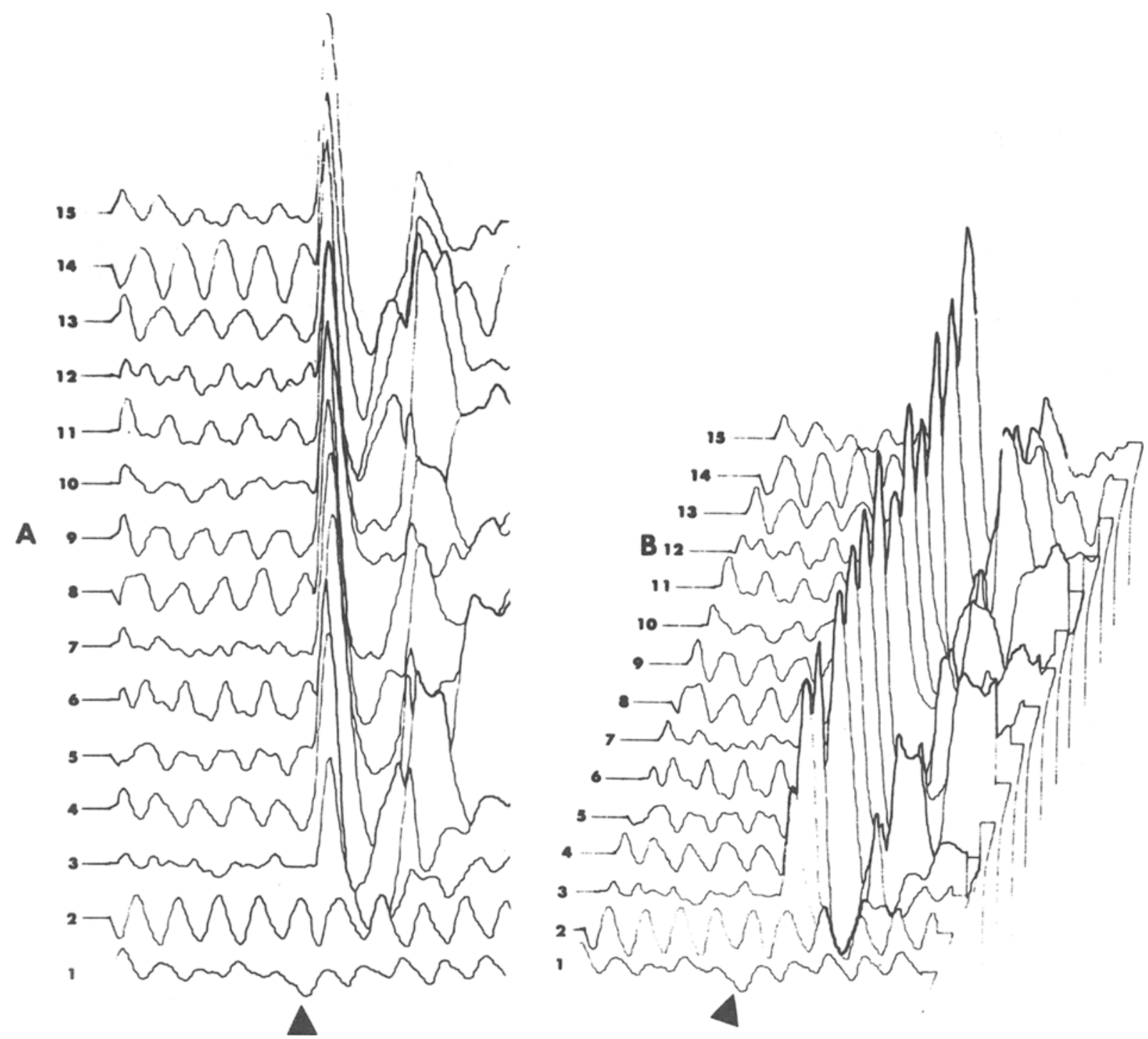

Fig. 2. Examples of plotting with hidden line suppression. The same data are plotted in A and B. A shows plots with no horizontal offset, while $B$ uses an offset of 10 units. Horizontal trace is 256 units.

digital to analog capabilities, as well as in assembly or computer languages.

(Received for publication June 4, 1973; accepted June 4, 1973.)

\section{CLUSTER: A hierarchical clustering program for large data sets $(n>100)$}

\section{JAY R. LEVINSOHN and SANDRA G. FUNK \\ L. 1. Thurstone Psychometric Laboratory University of North Carolina Chapel Hill, North Carolina 27514}

CLUSTER is a program especially designed to perform hierarchical clustering on large data matrices $(n>100)$. Although several excellent programs were already in existence for either single iteration or hierarchical procedures, none was oriented toward handling the massive data arrays often collected by current survey procedures in the behavioral and social sciences. Since clustering provides a good method for reducing large data arrays, a program specifically oriented toward such problems could be particularly useful.

CLUSTER is basically an extension of Veldman's (1967) HGROUP program. It utilizes Ward's (1963) hierarchical procedure of successively combining $n$ objects together to form $n, n-1, n-2, \cdots, 2$ groups by minimizing an objective error function. This error, or distance, function is defined to be the weighted sum of squared differences between corresponding scores on $m$ measures for each possible pairing of two groups. That 
is, given a matrix, $X$, of $m$ measures on $n$ objects, the initial error matrix is a $\mathrm{n} x \mathrm{n}$ symmetric matrix with the elements given by:

$$
\mathrm{d}_{\mathrm{ij}}=\sum_{\mathbf{k}=1}^{\mathrm{m}}\left(\mathrm{x}_{\mathrm{ik}}-\mathrm{x}_{\mathrm{jk}}\right)^{2} / 2 \quad \begin{aligned}
& \mathrm{i}=1,2, \cdots, \mathrm{n} \\
& \mathrm{j}=1,2, \cdots, \mathrm{n} .
\end{aligned}
$$

In existing clustering programs, it is not unusual for the entire error (distance) matrix to be stored in core. As data sets become large, core size is quickly exhausted. CLUSTER was designed to utilize a system of disk storage to accommodate these large matrices while maintaining the flexibility of smaller programs. Considerable leeway is allowed in the choice of core size and the number and size of disk records to be employed in order that even users of machines with small memory capacity may avail themselves of the CLUSTER program.

The program is coded in PL/l. It has been compiled and tested under both the IBM PL/F and IBM PL/O compilers using an IBM 370/165 under HASP/MVT. The program should run with no modification on any IBM $360 / 370$ using OS with at least a $100 \mathrm{~K}$ partition.

The program can handle data sets of virtually any size, perhaps limited only by time, funds, and available disk space. It is suggested that existing "in core" programs will be more efficient for processing smaller data arrays $(n<100)$.

In addition to the disk processing facilities previously mentioned, CLUSTER options allow:
(1) standardization of either rows or columns; (2) differential weighting of the measures; (3) clustering of either rows or columns; (4) a checkpoint/restart facility for run time control; and (5) backup files as a failsafe measure.

The user must supply (1) card input of the program parameters, and (2) either card or disk input of the raw data matrix or of a distance matrix if the raw data matrix is too large to fit in core.

On each of the $n-1$ iterations, the program outputs: (1) the number of groups remaining to be clustered on the next iteration; (2) the two groups combined on this step; (3) the number of members in these two groups; (4) the error incurred by this combination; and (5) the change in error over that of the previous iteration. Options allow the specification of the members of each group.

A user's guide, card or magnetic program source, and example runs may be obtained from Jay R. Levinsohn, L. L. Thurstone Psychometric Laboratory, University of North Carolina, Chapel Hill, North Carolina 27514.

\section{REFERENCES}

Veldman, D. J. Fortran programming. New York: Holt, Rinehart \& Winston, 1967.

Ward, J. H., Jr. Hierarchical grouping to optimize an objective function. Journal of the American Statistical Association.
$1963,58,236-244$.

(Received for publication Iuly 3, 1973; accepted July 8, 1973.) 